\title{
Two electrons in a strongly coupled double quantum dot: from an artificial helium atom to a hydrogen molecule
}

\author{
W. Dybalski* and P. Hawrylak \\ Institute for Microstructural Sciences, \\ National Research Council Canada, \\ Ottawa, Canada K1A0R6, \\ and \\ Physics Department, University of Ottawa, \\ 150 Louis Pasteur, Ottawa, Ontario K1N 6N5 Canada
}

(Dated: September 12, 2018)

\begin{abstract}
We study the formation of molecular states in a two-electron quantum dot as a function of the barrier potential dividing the dot. The increasing barrier potential drives the two electron system from an artificial helium atom to an artificial hydrogen molecule. To study this strongly coupled regime, we introduce variational wavefunctions which describe accurately two electrons in a single dot, and then study their mixing induced by the barrier. The evolution of the singlet-triplet gap with the barrier potential and with an external magnetic field is analyzed.

PACS numbers: 73.21.La, 03.67.Lx, 85.35.Be
\end{abstract}

\section{INTRODUCTION}

There is currently interest in developing means of isolating spins of individual electrons and coupling them in a controlled way $1,2,3,4,5,6,7,8.9$. This problem is equivalent to a formation and controlled dissociation of an artificial hydrogen molecule. This dissociation is not achieved by the increase of the separation of the hydrogen atoms, but by the increase of the tunneling barrier separating the dots. In such a process a single two-electron dot breaks into two one-electron dots. Hence the analogy to the break-up of the helium atom into the hydrogen molecule, or to nuclear fission, rather than to a chemical reaction. The description of the "artificial fission" process cannot be accomplished by the weak coupling approaches, such as the Hund-Mulliken method, and requires the treatment of a strongly coupled electron system. We develop such an approach in this work.

The quantum mechanical study of two electron atoms dates back to the work of Born and Heisenberg ${ }^{10}$ on the helium atom. (See ${ }^{11}$ for a review on two-electron atoms). Similar studies of artificial atoms followed the development of quantum dots 12.13 .14 - nanostructures in which the number of electrons can be reduced to a desired value $\left(n_{e}=0,1,2 \ldots\right)$ in a controllable manner. In contrast to atoms, the confining potential of a quantum dot is, to a good approximation, quadratic, so a dot containing one electron provides a realization of the exactly soluble Fock-Darwin model12.15.16.17.

The problem of two interacting electrons in a parabolic potential also admits exact solutions, but only for specific values of the oscillator frequency, as was shown by Taut $\frac{18.19 .20}{}$. The general case was treated analytically by the oscillator representation method 21 and variational calculations $^{22}$, and studied numerically by the following approaches: 'exact' diagonalization using Fock-Darwin states $^{23.24}$, integration of the radial motion Schroedinger equation after separating the center of mass motion ${ }^{25}$ and a combination of both ${ }^{26}$. The results were compared with experimental data ${ }^{24.26}$ and with the Hartree and HartreeFock methods 23 . The mean field approaches were applied to the two-electron system also in the context of symmetry breaking they may induce and subsequent symmetry restoration by $\mathrm{RPA}^{27}$ and projection techniques 28 . This theoretical problem, as well as the exact and numerical solutions mentioned above, proved relevant for a description of a Wigner molecule consisting of two electrons in a quantum $\operatorname{dot}^{29.30}$.

The problem of two vertically 31.32 .33 .34 .35 or laterally coupled dots $1,2,3.4,5.8$ containing one electron each is equivalent to the problem of an artificial hydrogen molecule. A variety of methods were applied here: The general case was studied by $\operatorname{LSDA}^{5}$, molecular orbital calculations ${ }^{3.4}$ and the Hartree-Fock approach ${ }^{4,28,36,37}$ refined subsequently by 'exact' diagonalization ${ }^{38}$ and projection techniques 28.36 .37 . The weakly coupled regime was studied analytically by the Heitler-London and Hund-Mulliken methods 2.3 . While the analytical results by the molecular Heitler-London and Hund-Mulliken approaches ${ }^{3}$ are very useful, the weakly coupled regime does not quantitatively describe the experimental situation. In molecular description the starting point are two well separated quantum dots. Then, as the distance between them is reduced, the electrons start tunneling from one dot to another in analogy to a chemical bond formation. However, in an actual experiment the double lateral dot is defined electrostatically by metallic gates located above the two dimensional electron gas 6.7 . Here the distance between the dots is held fixed and the coupling between them is controlled by means of the interdot barrier. When the barrier is zero the electrons move freely and our system is a single dot, an artificial helium atom. When the barrier increases, the single dot divides into two, the electrons reconfigure so as to avoid the barrier and an artificial hydrogen molecule forms. It is difficult to find an analogue of the above procedure in 
the realm of atomic physics. Nuclear physics, however, offers an obvious example - fission of a nucleus. In this work we demonstrate that viewing quantum dots as 'artificial nuclei' rather than 'artificial atoms' offers also some computational advantages.

Our paper is organized as follows: Section II describes our model consisting of a two-dimensional parabolic potential perturbed by a Gaussian barrier running along its diameter. In Subsection IIA we briefly discuss the exact eigenvectors of the single dot problem, found by Taut ${ }^{20}$, which are, however, correct only for specific values of the magnetic field, different for each state. In Subsection IIB we introduce variational wavefunctions which reduce to the exact eigenvectors at these specific magnetic fields. We calculate corresponding variational energies and com- pare them with exact and numerical values. In Section III we switch on the barrier and describe the formation of molecular states localized in the two potential minima, and the effect of the magnetic field on the singlet-triplet gap.

\section{THE MODEL HAMILTONIAN}

Our model Hamiltonian describes two electrons moving in the $(x, y)$ plane, confined by a parabolic potential with frequency $\omega_{0}$, perturbed by a Gaussian barrier of width $\Delta$ and hight $V_{0}$, and subject to a perpendicular magnetic field $\vec{B}$ :

$$
H=-\left(\vec{\nabla}_{1}+i \vec{A}\left(\vec{r}_{1}\right)\right)^{2}-\left(\vec{\nabla}_{2}+i \vec{A}\left(\vec{r}_{2}\right)\right)^{2}+\frac{1}{4} \omega_{0}^{2}\left(\vec{r}_{1}^{2}+\vec{r}_{2}^{2}\right)+\frac{2}{\left|\vec{r}_{1}-\vec{r}_{2}\right|}+V_{0}\left(e^{-\frac{x_{1}^{2}}{\Delta^{2}}}+e^{-\frac{x_{2}^{2}}{\Delta^{2}}}\right) .
$$

Here $\vec{A}\left(\vec{r}_{i}\right)=\left(\omega_{c} y_{i} / 4,-\omega_{c} x_{i} / 4,0\right), \omega_{c}=\frac{e B}{m^{*}}$ is the cyclotron frequency of an electron with effective mass $m^{*}$ and charge $-e$ placed in an external magnetic field $B$. The lengths are expressed in effective Bohr radii $a_{B}=\frac{4 \pi \epsilon \hbar^{2}}{m^{*} e^{2}}$ (where $\epsilon$ is the electric permeability), whereas $\omega_{0}, \omega_{c}$ and $V_{0}$ in effective rydbergs $\left(1 R y=\frac{\hbar^{2}}{2 m^{*} a_{B}^{2}}\right)$. The magnetic field points in the negative direction of the $z$ axis.

\section{A. Special exact solutions of the single dot problem}

In this and the following subsection we set $V_{0}=0$ and address the single dot problem. Change of variables $\vec{R}=\frac{\vec{r}_{1}+\vec{r}_{2}}{2}, \vec{r}=\vec{r}_{2}-\vec{r}_{1}$ separates the relative and center of mass motion in the Hamiltonian: $H=H_{R}+H_{r}$, where $H_{R}$ is the Fock-Darwin one-particle Hamiltonian. The detailed study of the radial Hamiltonian $H_{r}$ is presented in ${ }^{20}$. Here we only summarize the results which we will need in the sequel: The relative motion eigenfunction with angular momentum $m$ can be expressed as follows:

$$
\psi(\vec{r})=\sqrt[4]{\alpha} \frac{u_{m}(\rho)}{\sqrt{\rho}} \frac{e^{i m \phi}}{\sqrt{2 \pi}}
$$

where $\alpha=\frac{1}{4} \sqrt{\omega_{0}^{2}+\frac{\omega_{c}^{2}}{4}}, \rho=\sqrt{\alpha} r$. Aiming at the lowest excitations of the radial motion one obtains

$$
u_{m}(\rho)=C_{m} \rho^{\frac{1}{2}+|m|}\left(1+\sqrt{\frac{2}{1+2|m|}} \rho\right) e^{-\frac{\rho^{2}}{2}},
$$

(where $C_{m}$ are the normalization constants) and the corresponding eigenenergy:

$$
E_{m}=-\frac{\omega_{c} m}{2}+2 \frac{(2+|m|)}{1+2|m|} .
$$

However, the two expressions above are valid only if

$$
\alpha=\frac{1}{2(1+2|m|)} .
$$

In particular, for $m=0$ we get $E_{0}=4 R y$ provided that $\alpha=\frac{1}{2}$, and consequently $\omega_{c}=\omega_{c 0}=2 \sqrt{4-\omega_{0}^{2}}$. The corresponding wavefunction reads:

$$
u_{0}(\rho)=C_{0} \rho^{\frac{1}{2}}(1+\sqrt{2} \rho) e^{-\frac{\rho^{2}}{2}} .
$$

At zero magnetic field this is the ground state wavefunction, as it is nodeless. For $m= \pm 1$ the energy equals $E_{ \pm 1}=\mp \frac{\omega_{c} m}{2}+2 R y$, on condition that $\alpha=\frac{1}{6}$. This implies that $\omega_{c}=\omega_{c 1}=2 \sqrt{\frac{4}{9}-\omega_{0}^{2}}$. The triplet radial wavefunction follows:

$$
u_{1}(\rho)=C_{1} \rho^{\frac{3}{2}}\left(1+\sqrt{\frac{2}{3}} \rho\right) e^{-\frac{\rho^{2}}{2}} .
$$

For example, if $\omega_{0}=\frac{2}{3} R y$ then the triplets have the energy 2 Ry at zero magnetic field, whereas the lowest singlet has the energy 4 Ry at $\omega_{c}=\frac{8 \sqrt{2}}{3} \approx 3.77 R y$. But we are not able to get the exact energies at intermediate values of $\omega_{c}$. The subject of the next section will be a derivation of accurate upper bounds for these energies.

\section{B. Variational analysis}

To describe the lowest lying states of radial motion with arbitrary angular momentum we suggest variational 
wavefunctions inspired by the form of the eigenfunction (3). It does not seem reasonable to change the factors $\rho^{\frac{1}{2}+|m|}$ or $e^{-\frac{\rho^{2}}{2}}$ as it would spoil the behaviour of the function at zero or at infinity. The only remaining parameter is the one that multiplies $\rho$ in the bracket. Therefore, we introduce the following family of variational wavefunctions, labelled by the parameter $\beta$ :

$$
u_{m, \beta}(\rho)=C_{m, \beta} \rho^{\frac{1}{2}+|m|}(1+\beta \rho) e^{-\frac{\rho^{2}}{2}} .
$$

The corresponding variational energies follow:

$$
E_{m}(\beta)=-\frac{\omega_{c} m}{2}+2 \alpha \frac{a_{m}+b_{m} \beta+c_{m} \beta^{2}}{d_{m}+e_{m} \beta+f_{m} \beta^{2}},
$$

where $d_{m}=\frac{1}{2} \Gamma(1+|m|), e_{m}=\Gamma\left(\frac{3}{2}+|m|\right), f_{m}=\frac{1}{2} \Gamma(2+$ $|m|)$,

$$
\begin{aligned}
a_{m} & =\frac{e_{m}}{(2|m|+1) \sqrt{\alpha}}+2 f_{m}, \\
b_{m} & =\frac{2 d_{m}}{\sqrt{\alpha}}+2(|m|+1) e_{m}, \\
c_{m} & =\frac{e_{m}}{2 \sqrt{\alpha}}+\left(2 m^{2}+4|m|+3\right) d_{m} .
\end{aligned}
$$

The minimum $\beta_{m}$ of this simple function is found to be at one of the roots of the quadratic equation:

$$
\begin{array}{r}
\left(b_{m} d_{m}-a_{m} e_{m}\right)+2\left(c_{m} d_{m}-a_{m} f_{m}\right) \beta+ \\
\left(c_{m} e_{m}-b_{m} f_{m}\right) \beta^{2}=0 .
\end{array}
$$

There arises a question of accuracy of our method. To study this problem let us return to the example presented in the end of the previous subsection where $\omega_{0}=\frac{2}{3} R y \approx$ $4 \mathrm{meV}$ in GaAs. Clearly, our variational energies reproduce the exact eigenenergies at the specific values of $\omega_{c}$. (In this respect they improve upon the interpolation formula of Taut ${ }^{20}$.) To evaluate accuracy at intermediate values of $\omega_{c}$ we solve the radial eigenvalue problem numerically, by the Numerov method, and compare the results with our variational energies (Fig. 1). The picture shows that variational and numerical calculations are in very good agreement in a wide range of the magnetic field. The $m \neq 0$ energies calculated by both methods give almost identical results and are represented by single solid lines. The variational $m=0$ energy is even more accurate than the numerical result, as it is below the numerical value for large magnetic fields. Lower accuracy of the Numerov method is probably due to the fact that $m=0$ state is the only one which is non-zero in the singular point $\rho=0$.

In Fig. 11 we note the familiar singlet-triplet oscillations of the ground state 13.24 .25 .37 .40 . They are caused by a combination of two mechanisms: First, because of the orbital Zeeman term $-\frac{\omega_{c} L_{z}}{2}$ it is energetically favourable for the system to rotate. Nonetheless, it is clear that without interaction the ground state would be a singlet in any magnetic field. Second, Coulomb interaction acts stronger on singlets than on triplets, as in the latter the

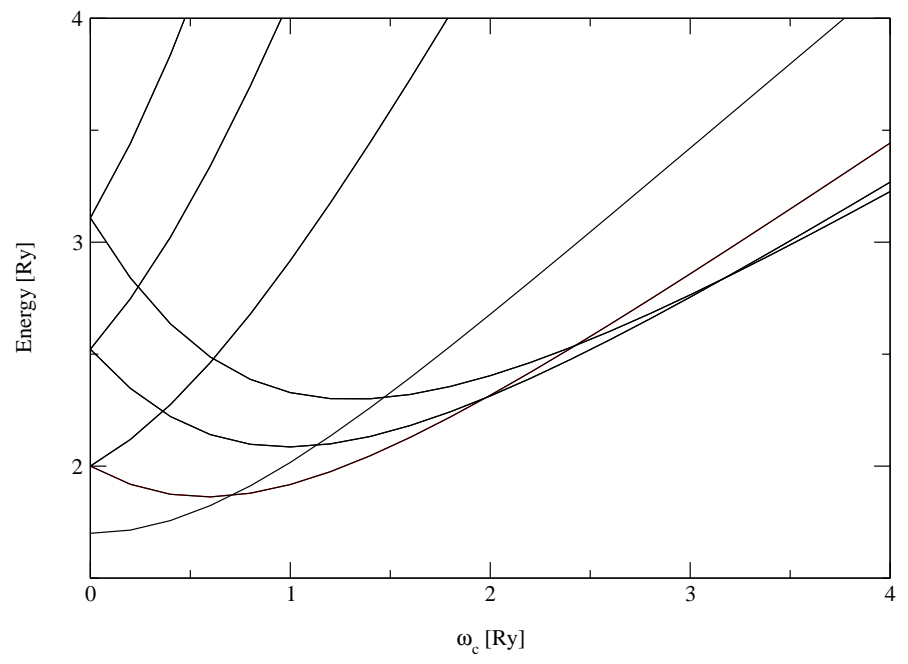

FIG. 1: Comparison of the relative motion energies in a parabolic dot with $\omega_{0}=\frac{2}{3} R y$ calculated variationally and by the Numerov method. Solid lines: variational $\mathrm{m}=0,1,-1,2$,$2,3,-3$ states (increasing order at $\omega_{c}=0.1 R y$ ). Dashed line: numerical $\mathrm{m}=0$ state. Other numerical states coincide with variational in this figure.

electrons are kept apart by the Pauli principle. Thereby, the gap between a singlet and a consecutive triplet is reduced below its non-interacting value. Nonetheless, without the Zeeman term the ground state is always a singlet no matter how strong is the interaction or the term quadratic in $\omega_{c}{ }^{39}$. We conclude that, in the presence of interaction, the increase of the magnetic field results in an increase of ground state angular momentum. As states with even $m$ are singlets, whereas states with odd $m$ are triplets, it causes singlet-triplet oscillations.

\section{DOUBLE DOT - FORMATION OF MOLECULAR STATES}

In this section we study the ground state and the first excited state energies and densities of two electrons in a double dot and their evolution with a magnetic field. For this purpose we go back to our model Hamiltonian (1) and set $V_{0}>0$. Clearly, the potential of the barrier:

$$
V_{b}=V_{0}\left(e^{-\frac{x_{1}^{2}}{\Delta^{2}}}+e^{-\frac{x_{2}^{2}}{\Delta^{2}}}\right)
$$

couples the motion of the center of mass and the relative motion. Nevertheless, we choose variational wavefunctions as products:

$$
\begin{aligned}
U_{m}(\rho, \varphi, \vec{R}) & =u_{m}(\rho, \varphi) \Psi_{0}(\vec{R}), \\
u_{m}(\rho, \varphi) & =u_{m, \beta_{m}}(\rho) \frac{e^{i m \varphi}}{\sqrt{2 \pi}},
\end{aligned}
$$


where the center of mass wavefunction is just the FockDarwin ground state:

$$
\Psi_{0}(\vec{R})=2 \sqrt{\frac{\alpha}{\pi}} e^{-2 \alpha R^{2}} .
$$

The corresponding energy of the center of mass motion is $E_{c m}=\sqrt{\omega_{0}^{2}+\frac{\omega_{c}^{2}}{4}}$. This choice is justified by the fact that the barrier couples only every second center of mass wavefunction as a result of parity conservation.
It is our goal to find matrix elements $H_{m, n}:=$ $\left\langle U_{m}|H| U_{n}\right\rangle$. To this end, we calculate the effective potential $V_{\text {eff }}(\rho, \varphi)=\left\langle\Psi_{0}\left|V_{b}\right| \Psi_{0}\right\rangle$ which acts only on the relative motion coordinates:

$$
V_{e f f}(\rho, \varphi)=\frac{2 V_{0} \gamma}{\sqrt{\gamma^{2}+1}} e^{-\frac{\rho^{2} \cos ^{2} \varphi}{\gamma^{2}+1}}
$$

where $\gamma=2 \Delta \sqrt{\alpha}$. Now we evaluate the matrix elements of the effective potential:

$$
\begin{array}{r}
\left\langle u_{m}\left|V_{\text {eff }}\right| u_{n}\right\rangle=V_{0} C_{m, \beta_{m}} C_{n, \beta_{n}} \frac{\gamma}{\sqrt{\gamma^{2}+1}}\left(K\left(\gamma^{2}+1, \frac{2+|m|+|n|}{2}, n-m\right)+\right. \\
\left.\left(\beta_{m}+\beta_{n}\right) K\left(\gamma^{2}+1, \frac{3+|m|+|n|}{2}, n-m\right)+\beta_{m} \beta_{n} K\left(\gamma^{2}+1, \frac{4+|m|+|n|}{2}, n-m\right)\right),
\end{array}
$$

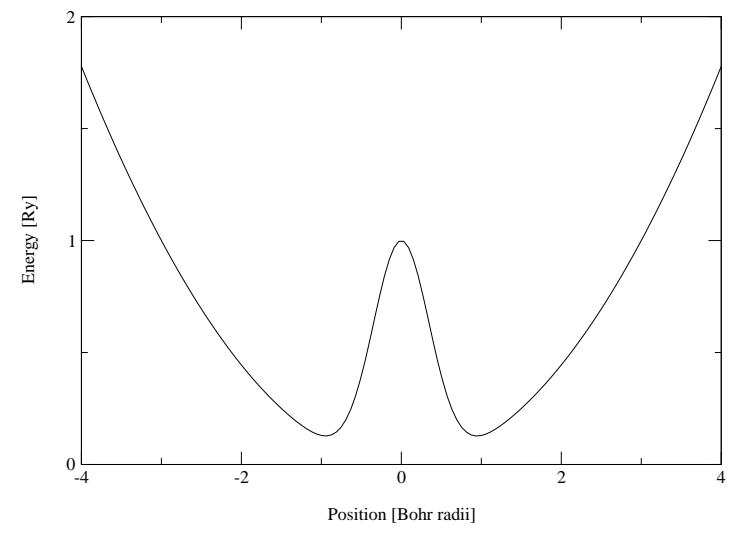

FIG. 2: The profile of the double dot potential with $\omega_{0}=$ $\frac{2}{3} R y, V_{0}=1 R y, \Delta=0.5 a_{B}$.

where $K(x, k, l)=\Gamma(k) \frac{1}{2 \pi} \int_{0}^{2 \pi} \frac{e^{i l \varphi}}{\left(1+\frac{1}{x} \cos ^{2} \varphi\right)^{k}} d \varphi$. For some values of $k, l$ this integral can be expressed in terms of elementary and elliptic functions. In practice, however, we evaluate it numerically. Finally, we have the desired expression for matrix elements of $H$ :

$$
H_{m, n}=\left(E_{m}\left(\beta_{m}\right)+E_{c m}\right) \delta_{m, n}+\left\langle u_{m}\left|V_{e f f}\right| u_{n}\right\rangle .
$$

We focus attention on the potential with parameters $\omega_{0}=\frac{2}{3} R y, V_{0}=1 R y, \Delta=0.5 a_{B}$. (The profile is shown in Fig. [2).

In the two subsections that follow we study the behaviour of the lowest lying singlet and triplet energies and densities at $0 \leq \omega_{c} \leq 1$. The third subsection describes the effect of the barrier on the singlet-triplet gap.

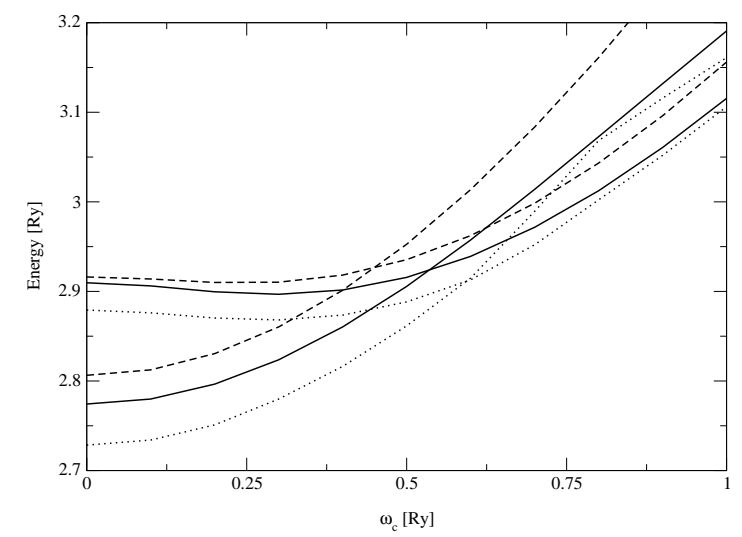

FIG. 3: The lowest lying singlet and triplet energies in a double dot with $\omega_{0}=\frac{2}{3} R y, V_{0}=1 R y, \Delta=0.5 a_{B}$. Dashed lines: $\tilde{E}_{0}$ - singlet, $\tilde{E}_{ \pm 1}$ - triplet. Solid lines: $\tilde{E}_{0, \pm 2}$ - singlet, $\tilde{E}_{ \pm 1, \pm 3}$ - triplet. Dotted lines: numerical singlet and triplet energies calculated by the configuration-interaction method. (In increasing order of energy at $\omega_{c}=0$ ).

\section{A. Energies and wavefunctions of singlets}

As a first approximation of the singlet energy we take only the $u_{0}$ state under consideration. The resulting energy:

$$
\tilde{E}_{0}=E_{0}\left(\beta_{0}\right)+E_{c m}+\left\langle u_{0}\left|V_{\text {eff }}\right| u_{0}\right\rangle
$$

is plotted as a function of the magnetic field in Fig. [3] and compared with numerical calculations performed using the configuration-interaction method.

Our variational energy is about $0.08 \mathrm{Ry}$ higher than the numerical one at $\omega_{c}=0$ and the discrepancy increases to $0.15 \mathrm{Ry}$ at $\omega_{c}=1 R y$. The reason for that is clear from Fig. 1] about $\omega_{c}=1 R y$ the $m=0$ state is close 
to degeneracy with $m=2$, therefore the latter should also be taken into account. On the other hand, $m=2$ state is degenerate with $m=-2$ at $\omega_{c}=0$ so, in fact, we should consider both of them. We diagonalize the resulting $3 \times 3$ matrix and note that its lowest eigenvalue $\tilde{E}_{0, \pm 2}$ differs from the numerical result by about 0.05 Ry at zero magnetic field (see Fig. 3) and this discrepancy becomes even smaller at larger $\omega_{c}$. We recall that $m \neq 0$ states vanish when the distance between the electrons is zero. Consequently, this increase in accuracy is the first manifestation of the formation of molecular states: contribution of $m= \pm 2$ states pushes the electrons apart and, by a non-trivial angular dependence, locates them in the dots. To support this statement we calculate the twoelectron density $\varrho$ in this approximation. Let us denote by $\Phi\left(\vec{r}_{1}, \vec{r}_{2}\right)$ the two-electron wavefunction. Then:

$$
\varrho\left(\vec{r}_{1}\right)=2 \int\left|\Phi\left(\vec{r}_{1}, \vec{r}_{2}\right)\right|^{2} d \vec{r}_{2} .
$$

We have calculated the wavefunction as a linear combination of functions $U_{m}(\rho, \varphi)$, with the coefficients $A_{m}$ found by numerical diagonalization. Recalling that $\rho=$ $\sqrt{\alpha} r, \vec{r}=(x, y)=\vec{r}_{2}-\vec{r}_{1}$ we obtain:

$$
\begin{array}{r}
\Phi\left(\vec{r}_{1}, \vec{r}_{2}\right)=\sum_{m} A_{m} C_{m, \beta_{m}} \frac{\sqrt{2}}{\pi} \alpha^{\frac{|m|+2}{2}}(x+i y)^{m} . \\
\cdot(1+\beta \sqrt{\alpha} r) e^{-\alpha\left(\vec{r}_{1}^{2}+\vec{r}_{2}^{2}\right)} .
\end{array}
$$

The integral (19) is evaluated numerically at $\omega_{c}=0 R y$ and $1 R y$. The results are compared with densities obtained by the configuration-interaction method. (See Fig. 4. Fig. [5). We note that the configuration-interaction calculations give a significantly lower density in the center of the dot than our variational method, especially at $\omega_{c}=0$. This can partly be attributed to the fact that we neglected the radial motion and center of mass excitations. The first excited state of radial motion with $m=0$ is close in energy to the $m= \pm 2$ states (see Fig. 10) and, in fact, lowers the density in the center of the dot as we show in the Appendix (see Fig. 11). On the other hand, the center of mass excitations which couple to the lowest singlet are separated by about $0.5 R y$ from $m= \pm 2$ states and we will not discuss them further.

Both methods predict that the electron density in the center of the double dot decreases with the magnetic field. Similarly, in the Heitler-London approach ${ }^{3}$ one observes that the overlap between the left and right dot wavefunction decreases as a function of $\omega_{c}$. From our perspective the explanation of this effect starts from the situation in a single dot. There, as the magnetic field increases, the lowest singlet has larger and larger angular momentum. Consequently, the density has a circular ring-like shape, with a minimum in the center, caused by the centrifugal barrier. Now, when the inter-dot barrier is switched on, the ring shrinks into two peaks as a result of mixing of angular momenta and radial excitations.

Summarizing our discussion in physical terms, the twoelectron droplet in the lowest singlet state acquires ro-
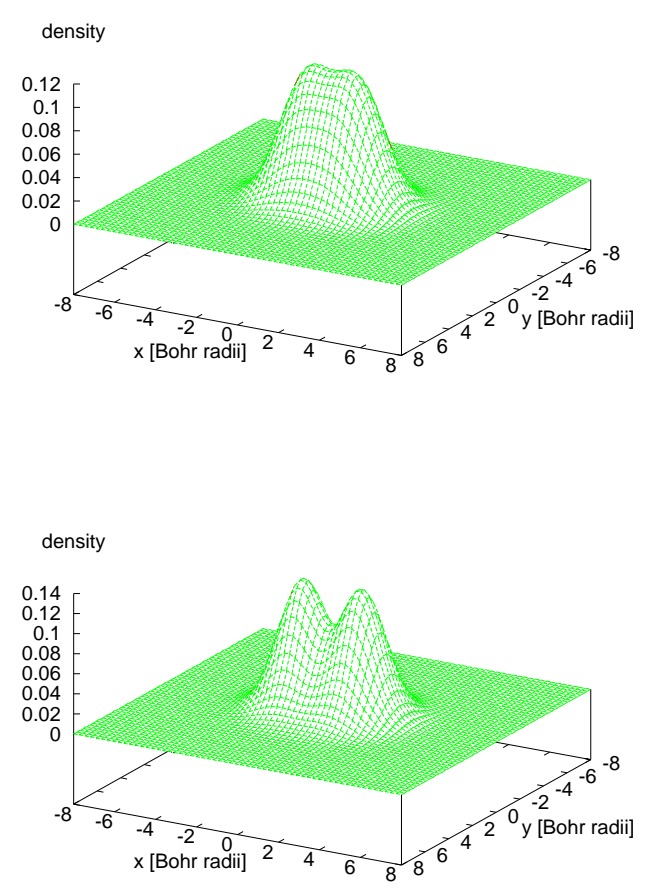

FIG. 4: The two-electron density of the lowest singlet calculated by diagonalization using $m=0, \pm 2$ states at $\omega_{c}=0 R y$ (upper fig.), $1 R y$ (lower fig.).
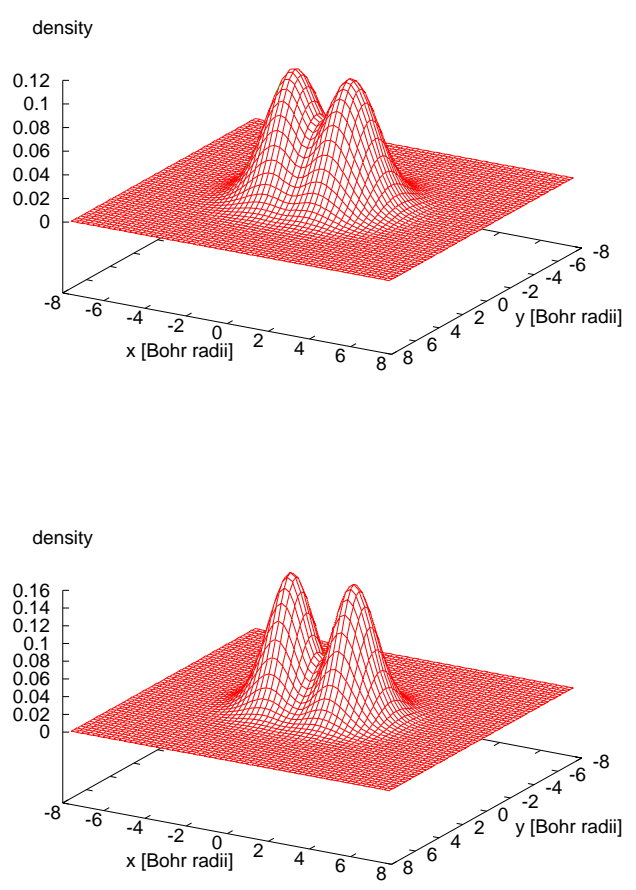

FIG. 5: The two-electron density of the lowest singlet calculated by the configuration-interaction method, at $\omega_{c}=0 R y$ (upper fig.), $1 R y$ (lower fig.). 

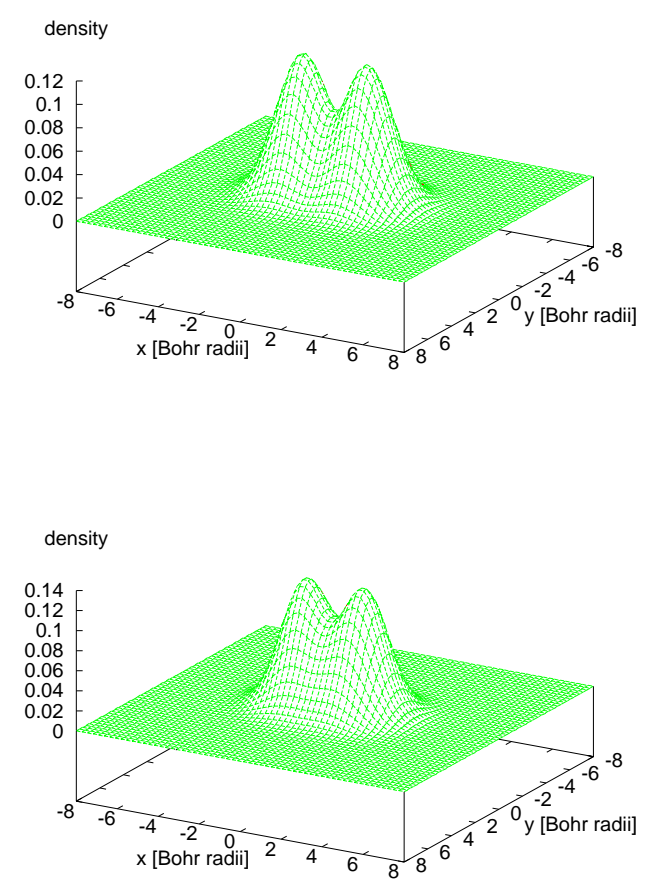

FIG. 6: The two-electron density of the lowest triplet calculated by diagonalization using $m= \pm 1, \pm 3$ states at $\omega_{c}=0 R y$ (upper fig.), 1 Ry (lower fig.).
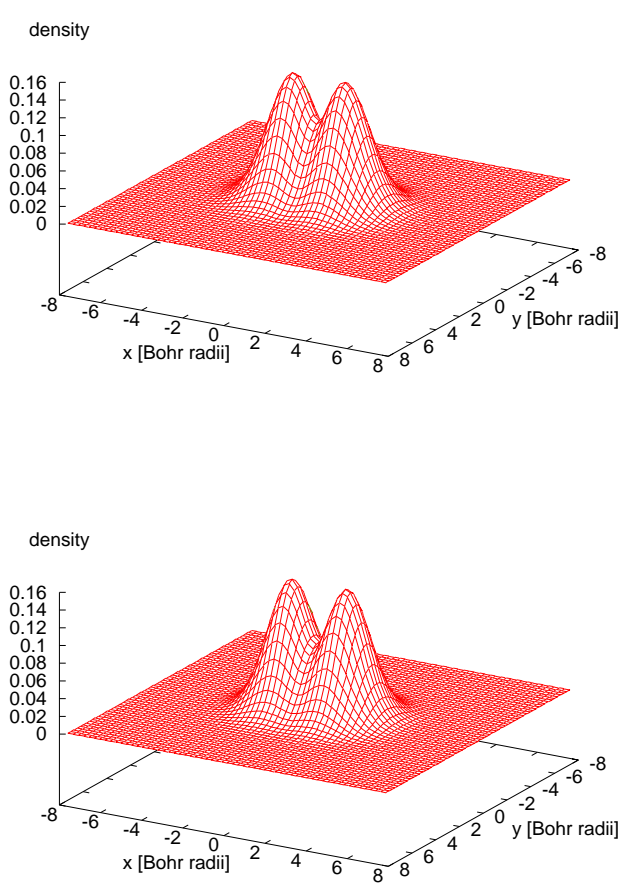

FIG. 7: The two-electron density of the lowest triplet calculated by the configuration-interaction method at $\omega_{c}=0 R y$ (upper fig.), $1 R y$ (lower fig.). tating and vibrating components, when the barrier is increased and a magnetic field applied. The vibrations have to be included at low magnetic fields to obtain qualitatively correct two-electron density. They counterbalance the non-rotating $(\mathrm{m}=0)$, peaked in the barrier, component of the droplet.

\section{B. Energies and wavefunctions of triplets}

Let us now describe the lowest lying triplet. First, we remark that the triplet energy, as a function of the magnetic field, will have a vanishing slope at $\omega_{c}=0$. In fact, breaking of circular symmetry will eliminate the degeneracy of $m= \pm 1$ states at $\omega_{c}=0$, making the wavefunction $\Phi$ of the lowest triplet real (up to a constant complex phase). Linear dependence of triplet energy could originate only from the orbital Zeeman term. But $\left\langle\Phi\left|L_{z} \otimes I+I \otimes L_{z}\right| \Phi\right\rangle=0$ for any real $\Phi$. Now we illustrate this general argument with a calculation of actual triplet energies taking only $m= \pm 1$ states under consideration. In this approximation the matrix elements of the Hamiltonian (17) read:

$$
\begin{aligned}
H_{1,1} & =E_{1}\left(\beta_{1}\right)+E_{c m}+\left\langle u_{1}\left|V_{e f f}\right| u_{1}\right\rangle, \\
H_{-1,-1} & =E_{-1}\left(\beta_{1}\right)+E_{c m}+\left\langle u_{-1}\left|V_{e f f}\right| u_{-1}\right\rangle, \\
H_{1,-1} & =\left\langle u_{1}\left|V_{\text {eff }}\right| u_{-1}\right\rangle .
\end{aligned}
$$

We recall from Subsection IIB that $E_{1}(\beta)=-\frac{\omega_{c}}{2}+F(\beta)$ and $E_{-1}(\beta)=\frac{\omega_{c}}{2}+F(\beta)$, where $F(\beta)=2 \alpha \frac{a_{1}+b_{1} \beta+c_{1} \beta^{2}}{d_{1}+e_{1} \beta+f_{1} \beta^{2}}$. They are both minimal for the same value of the variational parameter $\beta=\beta_{1}$ which solves the equation (10). The eigenvalues of the hermitian matrix defined by 21 . 23) are readily obtained :

$$
\begin{array}{r}
\tilde{E}_{ \pm}=F+E_{c m}+\left\langle u_{1}\left|V_{e f f}\right| u_{1}\right\rangle \pm \\
\sqrt{\frac{\omega_{c}^{2}}{4}+\left|\left\langle u_{1}\left|V_{e f f}\right| u_{-1}\right\rangle\right|^{2}} .
\end{array}
$$

The degeneracy of the states $m= \pm 1$ at zero field has been eliminated: there is a gap of $2\left|\left\langle u_{1}\left|V_{e f f}\right| u_{-1}\right\rangle\right|$. Moreover, the linear term $\pm \omega_{c} / 2$ is no longer present. Instead, there is a term $\sqrt{\frac{\omega_{c}^{2}}{4}+\left|\left\langle u_{1}\left|V_{e f f}\right| u_{-1}\right\rangle\right|^{2}}$ quadratic for small $\omega_{c}$. The lowest triplet energy $\tilde{E}_{-}=\tilde{E}_{ \pm 1}$ is plotted in Fig. 3. At zero field it is only about $0.4 R y$ higher than the numerical value. As the densities of $m= \pm 1$ states are not peaked in the barrier, it is not a surprise that the accuracy of our calculations is better than in the previous case. The discrepancy increases, however, with the magnetic field. To improve upon our approximation we take also $m= \pm 3$ states into account. The resulting energy $\tilde{E}_{ \pm 1, \pm 3}$ does not differ much from the previous one at $\omega_{c}=0$, but a remarkable accuracy was achieved at higher fields (see Fig. 3). As before, we plot electronic densities at $\omega_{c}=0 R y$ and $1 R y$. (See Fig. 6] Fig. 7). The low triplet density in the center of the double dot is inherited from the single dot. Loosely speaking, the 
two electron droplet in the lowest triplet state consists, from the outset, only of rotating components. After increasing the barrier and applying a magnetic field it will acquire components which rotate faster. As contrasted to the lowest singlet state, one can obtain a qualitatively valid description neglecting vibrations. This distinction between the lowest singlet and triplet states is likely to remain valid in more realistic double dots. It may find applications in spectroscopic measurements sensitive to charge distribution (e.g. in quantum point contact measurements).

\section{Singlet-triplet transition}

We define the singlet-triplet gap $J:=E_{t}-E_{s}$, where $E_{t}\left(E_{s}\right)$ denotes the energy of the lowest lying triplet (singlet) and plot the $J\left(\omega_{c}\right)$ dependence for a single dot $\left(\omega_{0}=\frac{2}{3} R y, V_{0}=0\right)$ in Fig. 8 Next, we move to the case of a double dot setting the parameters as in the previous subsections: $\omega_{0}=\frac{2}{3} R y, V_{0}=1 R y, \Delta=0.5 a_{B}$ and choose $E_{t}=\tilde{E}_{ \pm 1, \pm 3}, E_{s}=\tilde{E}_{0, \pm 2}$. The respective $J\left(\omega_{c}\right)$ function, plotted in Fig. 9] compares well with numerical results which are also presented.

In Section II we argued that the increase of the magnetic field results in an increase of ground state angular momentum leading to singlet-triplet oscillations of two electrons in a single dot. Although in a double dot the eigenstates do not have a definite angular momentum, the orbital Zeeman term and Coulomb interaction are still responsible for singlet-triplet transitions. In view of this fact and the discussion from the previous subsection on mixing of angular momenta, it is not a surprise that the barrier only smoothed out the sharp edges of the $J\left(\omega_{c}\right)$ function which were a direct consequence of angular momentum conservation. In particular, the positions of the first crossing and minimum are similar as without the barrier.

Since the precise shape of the dot does not play a role in the above discussion it seems to us possible that these qualitative features of the $J\left(\omega_{c}\right)$ dependence (the crossing, the minimum and the signature of the angular momentum conservation) are independent of the confining potential. (See e.g ${ }^{24}$ on elliptical dots). This claim is also reinforced by a recent analysis by Scarola and Das Sarma ${ }^{40}$ who related the singlet-triplet transitions to changes in vorticity of the two-electron wavefunction. Although their model potential and variational wavefunctions differ from ours, their results reflect the features mentioned above. Consequently, we expect that also realistic quantum dots of irregular shape will exhibit these properties. On the other hand, more detailed features, like the value of $J\left(\omega_{c}\right)$, the number of crossings or their precise positions will certainly depend on the shape of the dot.

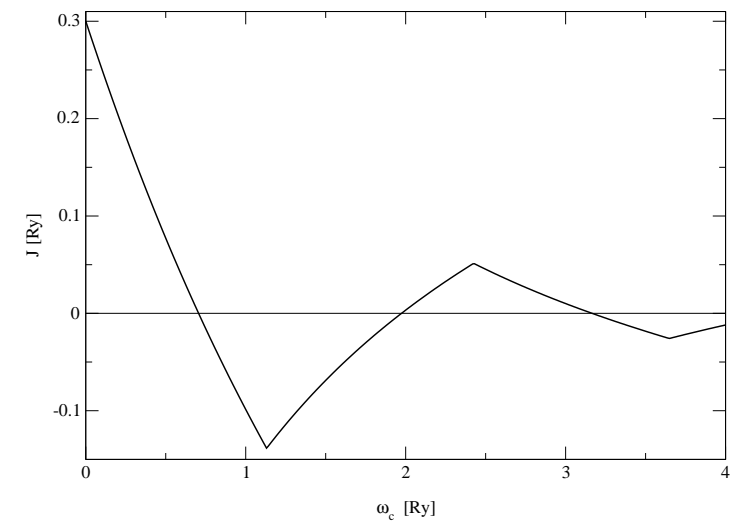

FIG. 8: The singlet-triplet gap as a function of the magnetic field in a single dot with $\omega_{0}=\frac{2}{3} R y$. Variational calculations.

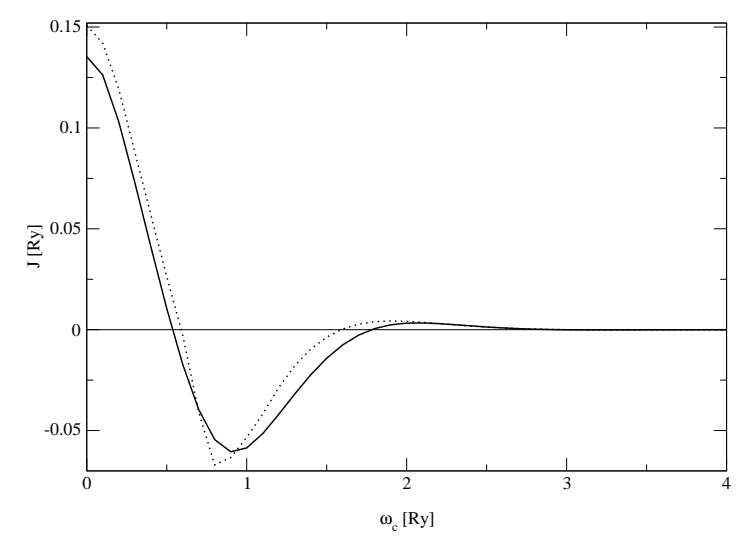

FIG. 9: The singlet-triplet gap as a function of the magnetic field in a double quantum dot with $\omega_{0}=\frac{2}{3} R y, V_{0}=1 R y, \Delta=$ $0.5 a_{B}$. Solid line: variational results. Dotted line: numerical calculations by the configuration-interaction method.

\section{CONCLUSION}

In this paper we discussed the problem of the transition from an artificial helium atom to a hydrogen molecule as a function of the barrier potential. We illustrated it with a simple calculation of the lowest lying singlet and triplet states in the strong coupling limit, where the Heitler-London approach is not valid. To achieve this goal we introduced variational wavefunctions which describe accurately two Coulomb interacting electrons in a parabolic quantum dot. The singlet and triplet energies, and the singlet-triplet gap $J$, were calculated as a function of the barrier potential and the magnetic field. The origin of the singlet-triplet transition was discussed. We hope that these variational functions will also be useful in developing methods of isolating spins of individual electrons and coupling them in a controllable manner in lateral quantum dots, as well as in other areas of research, e.g. to study with analytical expressions the formation 


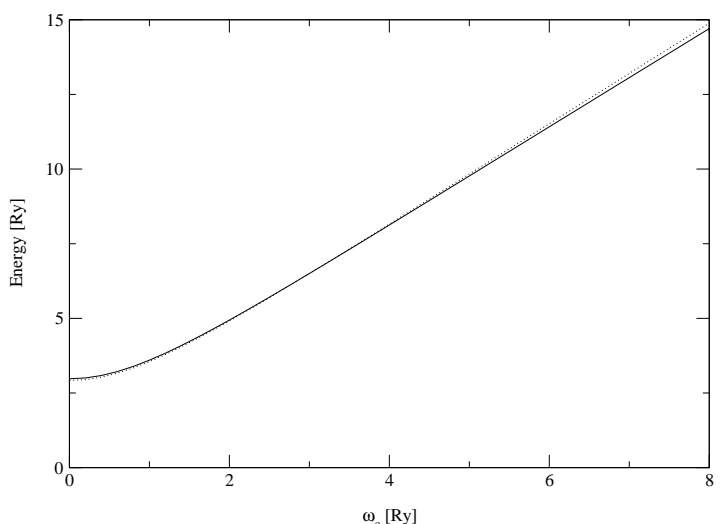

FIG. 10: The energy of the first excited state in a single parabolic dot with $\omega_{0}=\frac{2}{3} R y$. Solid line: variational calculation. Dotted line: numerical calculations by the Numerov method.

of Wigner molecules 29.30 .

We add two remark of a technical nature: First, in the case of a double quantum dot we were using the parameters $\beta$ optimized in a single circular dot. One could as well optimize them in the potential under study without much additional effort. It turns out, however, that the gain in accuracy is very small (at least in the case of the double dot presented here) so we did not pursue this approach. Second, a more realistic model of a double dot would be a single elliptical dot perturbed by a barrier. Since an elliptical confinement still separates the center of mass and relative motion it is not difficult to adapt our method to this case. Quantitative agreement with numerical results would require, however, more effort as our variational wavefunctions are optimized in a circular dot. On the other hand, our approach works well in the case of a single elliptical dot (no barrier) or a quantum ring (a single circular dot with a circular barrier).

\section{Acknowledgments}

The authors acknowledge support by the Institute for Microstructural Sciences, NRC, by the National Science and Engineering Research Council, and by the Canadian Institute for Advanced Research. One of the authors (W.D.) also acknowledges support from the EC Research Training Network 'Quantum Spaces - Non-commutative Geometry'. The authors thank M. Pioro-Ladriere, A. S. Sachrajda, M. Abolfath, C. Dharma-wardana, and W. Jakobiec for stimulating discussions during the course of this work.

\section{APPENDIX: THE EFFECT OF RADIAL EXCITATIONS ON THE DENSITY IN A DOUBLE DOT}

In this Appendix we calculate the two-electron density of the lowest singlet in a double dot taking into account the first excited state of the radial motion with $m=0$. To this end, we proceed as follows: We start from a certain exact solution of the single dot problem. Then we construct a variational wavefunction of a similar functional form and orthogonalize it to the ground state variational wavefunction. Finally, we diagonalize the double dot Hamiltonian using the excited state $m=0^{*}$ together with the previously studied $m=0, m=2, m=-2$ states.

From the analysis by Taut ${ }^{20}$ we obtain that at $\alpha=\frac{1}{12}$ there is an eigenstate of energy $E=1 R y$ given by:

$$
\tilde{u}_{0}(\rho)=\tilde{C}_{0} \rho^{\frac{1}{2}}\left(1+\sqrt{12} \rho+2 \rho^{2}\right) e^{-\frac{\rho^{2}}{2}} .
$$

As a matter of fact, at zero magnetic field it is the ground state, since it is positive. But the binding potential corresponding to this eigenstate is much weaker than the one we have under study. We expect that at a stronger binding potential the first excited state with $m=0$ will have such functional form so we describe it by a variational wavefunction:

$$
\tilde{u}_{0, \tilde{\beta}}(\rho)=\tilde{C}_{0, \tilde{\beta}} \rho^{\frac{1}{2}}\left(1+\tilde{\beta} \rho+\tilde{\delta} \rho^{2}\right) e^{-\frac{\rho^{2}}{2}},
$$

where $\tilde{C}_{0, \tilde{\beta}}$ is a normalization constant, $\tilde{\beta}, \tilde{\delta}$ are variational parameters. First of all, we have to make sure that the state is orthogonal to $u_{0, \beta_{0}}$ :

$$
\begin{array}{r}
0=\int_{0}^{\infty} \tilde{u}_{0, \tilde{\beta}}(\rho) u_{0, \beta_{0}}(\rho) d \rho= \\
\frac{1}{2}\left(1+\frac{1}{2} \sqrt{\pi}\left(\beta_{0}+\tilde{\beta}\right)+\left(\tilde{\delta}+\beta_{0} \tilde{\beta}\right)+\frac{3}{4} \sqrt{\pi} \tilde{\delta} \beta_{0}\right) .
\end{array}
$$

This implies that $\tilde{\delta}=-\Delta_{1}-\Delta_{2} \tilde{\beta}$, where

$$
\begin{aligned}
\Delta_{1} & =\frac{1+\frac{1}{2} \sqrt{\pi} \beta_{0}}{1+\frac{3}{4} \sqrt{\pi} \beta_{0}}, \\
\Delta_{2} & =\frac{\frac{1}{2} \sqrt{\pi}+\beta_{0}}{1+\frac{3}{4} \sqrt{\pi} \beta_{0}} .
\end{aligned}
$$

Next, we evaluate the normalization constant:

$$
\begin{aligned}
\tilde{C}_{0, \tilde{\beta}}^{-2} & =\tilde{d}+\tilde{e} \tilde{\beta}+\tilde{f} \tilde{\beta}^{2}, \\
\tilde{d} & =\Delta_{1}^{2}-\Delta_{1}+\frac{1}{2}, \\
\tilde{e} & =\frac{1}{2} \sqrt{\pi}-\frac{3}{4} \sqrt{\pi} \Delta_{1}+2 \Delta_{1} \Delta_{2}-\Delta_{2}, \\
\tilde{f} & =\frac{1}{2}-\frac{3}{4} \sqrt{\pi} \Delta_{2}+\Delta_{2}^{2} .
\end{aligned}
$$

Now we are ready to calculate the variational energy: 


$$
\begin{aligned}
\tilde{E}_{0}(\tilde{\beta}) & =2 \alpha \int_{0}^{\infty} \tilde{u}_{0, \tilde{\beta}}(\rho)\left\{-\partial_{\rho}^{2}+\left[\frac{1}{\sqrt{\alpha} \rho}+\rho^{2}-\frac{1}{4 \rho^{2}}\right]\right\} \tilde{u}_{0, \tilde{\beta}}(\rho) d \rho, \\
\tilde{E}_{0}(\tilde{\beta}) & =2 \alpha \frac{\tilde{a}+\tilde{b} \tilde{\beta}+\tilde{c} \tilde{\beta}^{2}}{\tilde{d}+\tilde{e} \tilde{\beta}+\tilde{f} \tilde{\beta}^{2}}, \\
\tilde{a} & =\left(\frac{1}{2} \sqrt{\frac{\pi}{\alpha}}+1\right)-2 \Delta_{1}\left(\frac{1}{4} \sqrt{\frac{\pi}{\alpha}}+1\right)+\Delta_{1}^{2}\left(\frac{3}{8} \sqrt{\frac{\pi}{\alpha}}+4\right), \\
\tilde{b} & =\left(\frac{1}{\sqrt{\alpha}}+\sqrt{\pi}\right)-2 \Delta_{2}\left(1+\frac{1}{4} \sqrt{\frac{\pi}{\alpha}}\right)-\Delta_{1}\left(\frac{5}{2} \sqrt{\pi}+\frac{1}{\sqrt{\alpha}}\right)+2 \Delta_{1} \Delta_{2}\left(4+\frac{3}{8} \sqrt{\frac{\pi}{\alpha}}\right), \\
\tilde{c} & =\left(\frac{1}{4} \sqrt{\frac{\pi}{\alpha}}+\frac{3}{2}\right)-\Delta_{2}\left(\frac{5}{2} \sqrt{\pi}+\frac{1}{\sqrt{\alpha}}\right)+\Delta_{2}^{2}\left(4+\frac{3}{8} \sqrt{\frac{\pi}{\alpha}}\right) .
\end{aligned}
$$

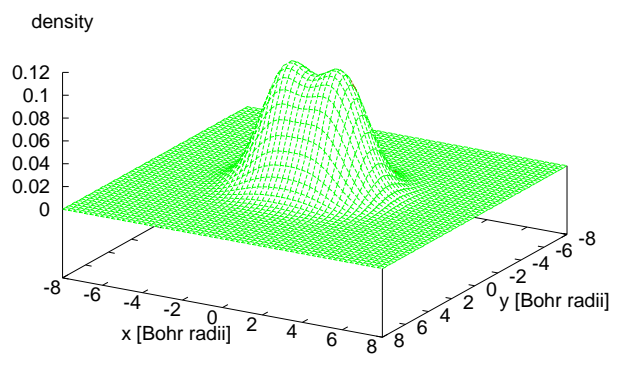

The optimal variational parameter $\tilde{\beta}_{0}$ can be found by solving a quadratic equation analogous to (10). The energy is then obtained substituting it back to equation (35). At this stage a comparison can be made between our variational approach and a numerical solution of the single dot problem by the Numerov method. The results are plotted in Fig. 10] Similarly as in Section II, we note very good agreement between the two methods. Finally, we arrive at a task of computing matrix elements of the effective potential $V_{\text {eff }}$ :

FIG. 11: The two-electron density at $\omega_{c}=0 R y$ calculated using $m=0, m=0^{*}, m=2, m=-2$ states.

$$
\begin{aligned}
\left\langle\tilde{u}_{0, \tilde{\beta}_{0}}\left|V_{\text {eff }}\right| \tilde{u}_{0, \tilde{\beta}_{0}}\right\rangle= & \frac{V_{0} \gamma \tilde{C}_{0, \tilde{\beta}_{0}}^{2}}{\sqrt{\gamma^{2}+1}}\left(K\left(\gamma^{2}+1,1,0\right)+\left(\tilde{\beta}_{0}^{2}+2 \tilde{\delta}\right) K\left(\gamma^{2}+1,2,0\right)+\right. \\
& \left.\tilde{\delta}^{2} K\left(\gamma^{2}+1,3,0\right)+2 \tilde{\beta}_{0} K\left(\gamma^{2}+1, \frac{3}{2}, 0\right)+2 \tilde{\beta} \tilde{\delta} K\left(\gamma^{2}+1, \frac{5}{2}, 0\right)\right), \\
\left\langle\tilde{u}_{0, \tilde{\beta}_{0}}\left|V_{e f f}\right| u_{m}\right\rangle= & \frac{V_{0} \gamma \tilde{C}_{0, \tilde{\beta}_{0}} C_{m, \beta_{m}}}{\sqrt{\gamma^{2}+1}}\left(K\left(\gamma^{2}+1, \frac{2+|m|}{2}, m\right)+\left(\beta_{m}+\tilde{\beta}_{0}\right) K\left(\gamma^{2}+1, \frac{3+|m|}{2}, m\right)+\right. \\
& \left.\left(\tilde{\beta}_{0} \beta_{m}+\tilde{\delta}\right) K\left(\gamma^{2}+1, \frac{4+|m|}{2}, m\right)+\tilde{\delta} \beta_{m} K\left(\gamma^{2}+1, \frac{5+|m|}{2}, m\right)\right) .
\end{aligned}
$$

(The function $K$ was defined in Section III.) Together with matrix elements from Section III we have all input necessary to diagonalize the double dot Hamiltonian in the subspace spanned by the $m=0, m=0^{*}, m=2, m=$ -2 states (where $0^{*}$ denotes the first excited state with $m=0$, determined in this Appendix). Having obtained the wavefunction, we calculate the density at $\omega_{c}=0$ and plot it in Fig. 11] Comparison with the density at $\omega_{c}=0$ calculated previously (Fig. (4) indicates that the radial motion excitation lowers the density in the center of the double dot. 
* Present address: Institut für Theoretische Physik, Universität Göttingen, Friedrich-Hund-Platz 1, D-37077 Göttingen - Germany.

1 J. A. Brum and P. Hawrylak, Superlatt. Microstruct. 22, 431 (1997).

2 D. Loss and D. P. DiVincenzo, Phys. Rev. A 57, 120 (1998).

3 G. Burkard, D. Loss and D. P. DiVincenzo, Phys. Rev. B 59, 2070 (1999).

4 X. Hu and S. Das Sarma, Phys. Rev. A 61, 062301 (2000).

5 A. Wensauer, O. Steffens, M. Suhrke and U. Rossler, Phys. Rev. B 62, 2605 (2000).

6 M. Pioro-Ladriere,M. Ciorga, J. Lapointe, P. Zawadzki, M. Korkusinski, P. Hawrylak, A. S. Sachrajda, Phys. Rev. Lett. 91, 026803 (2003).

7 W. G. van der Wiel, S. De Franceschi, J.M. Elzerman, T. Fujisawa, S. Tarucha and L.P. Kouwenhoven, Rev. Mod. Phys. 75, 1 (2003).

J. M. Elzerman, R. Hanson, J. S. Greidanus, L. H. Willems van Beveren, S. De Franceschi, L. M. K. Vandersypen, S. Tarucha and L. P. Kouwenhoven, Physica E 25, 135 (2004).

8 L.-X. Zhang, P. Matagne, J. P. Leburton, R. Hanson, and L. P. Kouwenhoven, Phys. Rev. B 69, 245301 (2004).

${ }^{9}$ M. Pioro-Ladriere, R. Abolfath, P. Zawadzki, J. Lapointe, S. A. Studenikin, A. S. Sachrajda, P. Hawrylak, cond-mat/0504009 v1 (2005).

10 M. Born and W. Heisenberg, Z.Phys. 16, 229 (1923).

11 H. A. Bethe, Quantum Mechanics of One-And TwoElectron Atoms, Plenum Publishing Corporation 1977.

12 L. Jacak, P. Hawrylak and A. Wojs, Quantum Dots, Springer, Berlin 1997.

13 R. C. Ashoori, Nature 379, 413 (1996).

14 S. M. Reimann and M. Manninen, Rev. Mod. Phys 74, 1283 (2002).

15 V. Fock, Z. Phys. 47, 446 (1928).

16 C. G. Darwin, Proc. Cambridge Philos.Soc. 27, 86 (1930).

17 S. Raymond, S. Studenkin, A. Sachrajda, Z. Wasilewski, S. J. Cheng, W. Sheng, P. Hawrylak, A. Babinski, M. Potemski, G. Ortner, M. Bayer, Phys. Rev. Lett. 92, 187402 (2004).

18 M. Taut, Phys. Rev. A 48, 3561 (1993).

19 M. Taut, A. Ernst and H. Eschrig, J. Phys. B. 31, 2689 (1998).
20 M. Taut, J. Phys. A: Math. Gen. 27, 1045 (1994).

21 M. Dineykhan and R. G. Nazmitdinov, Phys. Rev. B 55, 13707 (1997)

22 S. Das, P. Goswami, J. K. Bhattacharjee, cond-mat/0201225 v1 (2002).

23 D. Pfannkuche, V. Gudmundsson and P. A. Maksym, Phys. Rev. B 47, 2244 (1993).

24 J. Kyriakidis, M. Pioro-Ladriere, M. Ciorga, A. S. Sachrajda and P. Hawrylak, Phys. Rev. B 66, 035320 (2002).

25 M. Wagner, U. Merkt and A. V. Chaplik, Phys. Rev. B 45, R1951 (1992).

${ }^{26}$ P. Hawrylak, Phys. Rev. Lett. 71, 3347 (1993).

27 L. Serra, R. G. Nazmitdinov and A. Puente, Phys. Rev. B 68, 035341 (2003).

28 C. Yannouleas and U. Landman, J. Phys.: Condens. Matter 14L., 591 (2002).

29 A. Puente, L. Serra and R. G. Nazmitdinov , Phys. Rev. B, 69, 125315 (2004).

30 C. Yannouleas and U. Landman, Phys. Rev. Lett. 85, 1726 (2000).

31 J. J. Palacios and P. Hawrylak, Phys. Rev. B 51, 1769 (1995)

32 M. Bayer, P. Hawrylak, K. Hinzer, S. Fafard, M. Korkusinski, Z. R. Wasilewski, O. Stern, A. Forchel, Science 291, 451 (2001).

33 M. Rontani, S. Amaha, K. Muraki, F. Manghi, E. Molinari, S. Tarucha, D. G. Austing, Phys. Rev. B, 69, 085327 (2004).

34 E. Anisimovas and F. M. Peeters, Phys. Rev. B, 65, 233302 (2002).

35 R. Ravishankar, P. Matagne, J. P. Leburton, R. M. Martin, S. Tarucha Phys. Rev. B, 69, 035326 (2004).

${ }^{36}$ C. Yannouleas and U. Landman, Eur. Phys. J. D 16, 373 (2001)

37 C. Yannouleas and U. Landman, Int. J. Quantum Chem. 90, 699 (2002).

38 X. Hu and S. Das Sarma, Phys. Rev. A 64, 042312 (2001).

39 M. Read and B. Simon, Methods of Modern Mathematical Physics vol.4, Academic Press, New York, 1978.

40 V. W. Scarola and S. Das Sarma, Phys. Rev. A 71, 032340 (2005) 